(C) 2016 IEEE. Personal use of this material is permitted. Permission from IEEE must be obtained for all other uses, in any current or future media, including reprinting/republishing this material for advertising or promotional purposes, creating new collective works, for resale or redistribution to servers or lists, or reuse of any copyrighted component of this work in other works. 


\section{Split square resonators using high dielectric constant resonator}

\author{
B. Moradi, U. Matrinez and J. Garcia
}

This paper reports the design of a split ring resonator band-pass filter. The design is based on coupling coefficient filter and the novelty of the proposed design is possibility to miniaturize the structure by including a new kind of dielectric resonator fabricated with a commercial high dielectric constant EPOXY paste. Equivalent circuit model is discussed, establishing a clear relation between each component and physical parameter of the proposed design. Excellent agreement between electromagnetic simulation and measured of band pass filter at 1.65 $\mathrm{GHz}$ is found.

Introduction: The miniaturization of resonant particles has become interesting field after emerge of Split Ring Resonator (SRR) based metamaterial (MTM) in 1999 by Pendry [1]. The sub-wavelength property of the SRR has been successfully applied to the miniaturization of passive microwaves devices [2]. Different approaches by using coupling principle have been used to design compact resonators such as the Non-Bianisotropic SRR (NB-SRR), the Broad Side Coupling SRR (BCSRR) [3] or even more complicate shapes [1]. These resonant particles are theoretically suitable for MTMs designs, however, due to the degradation of the quality factor (Q) and the ohmic loses the practical applications exhibit a very low performance [4]. On the other hand, the presence of very close resonances in the vicinity of the subwavelength resonances produces spurious bands in the designs based on these resonators that complicate their even more than their practical applications [5]. The geometry of the proposed split square resonator (SSR) is depicted in Fig. 1. The square shape of the resonators has been chosen to maximize the capacitance of the LC resonant tank, resulting in a final thickness of $3.1 \mathrm{~mm}$, and to maximize the coupling between resonators according with the previous experience with this kind of design [2], [6]. The minimal distance between the resonators is $0.2 \mathrm{~mm}$ and the distance between the port line and the resonators has been fixed to $0.15 \mathrm{~mm}$ which is the more critical distance of the design. The ports feed the resonators though a capacitive coupling which intensity depends of the proximity to the extreme resonators $(1.5 \mathrm{~mm})$.

The input and output ports are $50 \Omega$ micro-strip lines that in this substrate correspond to $1.6 \mathrm{~mm}$ thick microstrip. Extensions of the ports with a $90^{\circ}$ angle following the square resonator profile have been included in order to maximize the coupling with the side resonators. The fact that there are dimensions in the layout of the order of the metallic layer thickness requires the utilization of an electromagnetic simulator able to have into account this thickness.

Equivalent circuit model of SSR band pass filter: The equivalent circuit model of a filter helps to understand the behaviour of the design. In the case in which a clear relationship between the equivalents circuit model and the layout physical dimensions can be set. Fig. 2 shows the equivalent circuit model of the proposed filter. Basically it is composed by three LC resonant parallel tanks that represent the three open rings, and a set of capacitive coupling networks to model the different couplings. The nature of the coupling may be magnetic or electric and, in general, it uses to be a combination of both. In many cases, the geometry symmetries can force the predominance of one of the couplings [5].

In our case the orientation of the central resonator has been inverted respect to the orientation of the extremes resonators in such a way that the current distribution in the resonances guarantees the existence of a magnetic wall between adjacent particles and therefore the coupling is forced to be mainly capacitive.

As can be observed from Fig. 2 there are three different kinds of coupling, characterized by the parameters $C_{P}, C_{M}$ and $C_{K} \cdot C_{P}$ is modelling the coupling between the port extensions and the extern resonators. The value of this parameter can be modified in the layout altering the horizontal distance between the port extension and the adjacent resonator. Also, there is a dependence of this parameter with the side of the adjacent resonator, being possible to modify this coupling using rectangular shape resonators. The $\mathrm{C}_{\mathrm{K}}$ parameter models the coupling between adjacent resonators and can be controlled directly with the distance between the resonators.

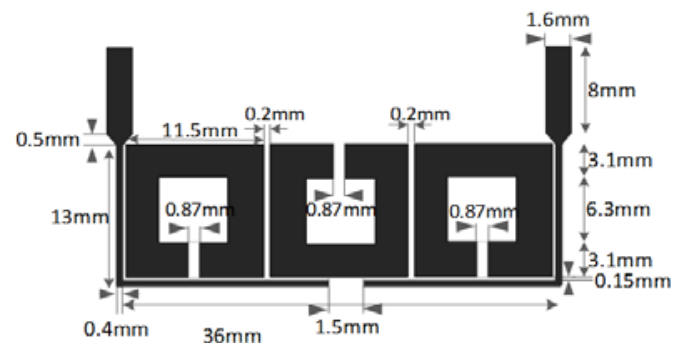

Fig. 1 Dimensional layout of the proposed filter.

The maximum value of this capacitance is fixed by the minimal distance between metallic layers that the fabrication technology allows. This value is important because, according with the expressions that link the coupling coefficient and the standard normalized filter parameters [7], the maximum bandwidth of the filters is bounded to the maximum coupling coefficient. Finally, $\mathrm{C}_{\mathrm{M}}$ represents the cross coupling coefficient that represent the novelty of the proposed design. The equivalent circuit model of the capacitive coupling is a $\Pi$ circuit with two negative capacitors in the parallel branches. Interpreting the negative capacitors as inductors, the structure can be thought as L-C-L matching ladder. Since the value of the $C_{M}$ can be controlled with the variation of the overlap between the port extension and the central square resonator, this matching ladder can be tuned in a certain range of values. The equivalent circuit model proposed is based on the description of the capacitive coupling coefficient as a $\Pi$ model. The values of the different parameters can be initially estimated from the physical dimensions, but at the end there will be necessary to optimize them to fit the measured response.

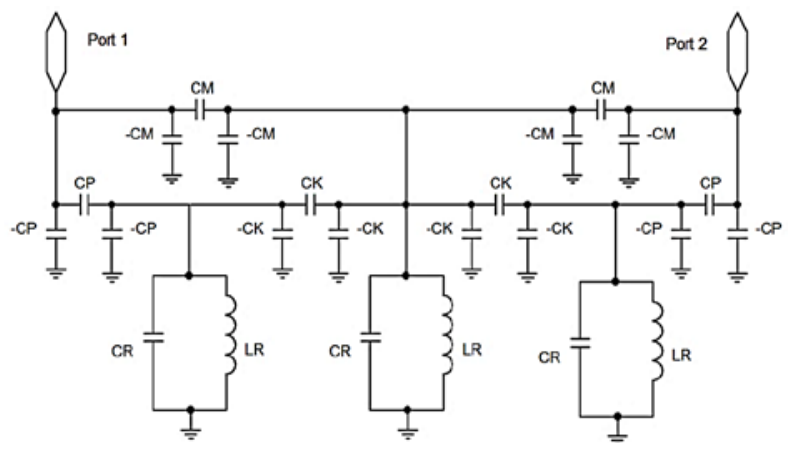

Fig. 2 Equivalent circuit model of the proposed filter.

Fabrication process and measured result: SSR band pass filter is implemented by using a Protolaser milling machine in a 50 mil Rogers RO3010 substrate which is characterized by a loss tangent $\delta=0.0022$ and $\varepsilon_{\mathrm{r}}=10.2$. The minimal distance between the resonators is $0.2 \mathrm{~mm}$ and the distance between the port line and the resonators has been fixed to $0.15 \mathrm{~mm}$ which is the more critical distance of the design. The photograph of the fabricated proposed filter is depicted in Fig. 3a. There is an excellent agreement in the resonant frequencies position is observed between simulation, measurement and equivalent circuit model as depicted in Fig $3 b$. There is a band pass between $1.5 \mathrm{GHz}$ and $1.8 \mathrm{GHz}$ which is performing a $18.1 \%$ bandwidth. The return losses are below the $-10 \mathrm{~dB}$ in all the band pass and the insertion losses oscillate between $-1.98 \mathrm{~dB}$ and $0.79 \mathrm{~dB}$.

Miniaturization of the filter by embedded DR: One of the more interesting properties of the proposed design is the possibility to miniaturize the structure by including high dielectric constant in the center of the SSR [8]. The embedded dielectric resonators (EDR) is produced by depositing high dielectric constant paste in a cavity milled in the substrate.

By drilling an array of square via-slot patterns in a substrate, waveguide dielectric channel can be created. Layers of the epoxy have been deposited until a thickness of the substrate has been achieved in the 
resonators. There are two main advantage of this technique to generate EDRs: the possibility to control the geometry of the resonator and the possibility to combine with standard structures in planar technologies such as microstrip or coplanar-waveguide. Epoxy dielectric material is used as DRs. A drying process is needed to obtain the EDR however the procedure is compatible with low LTCC (Low Temperature Co-Fired Ceramics) technology. The design miniaturization level increases with the value of the dielectric constant of the EDR. The EDR cavity is milled inside the three ring space as can be observed in Fig. 4. Dielectric paste with $\varepsilon_{\mathrm{r}}=45$ has been deposited into the cavity and the structure has been dried in a conventional oven at $150^{\circ} \mathrm{C}$ for one hour. The EDR presented in this work constitutes a new approach to the miniaturized resonators suitable for MTMs design without the $Q$ degradation inherent to the coupling coefficient based sub-wavelength particles.

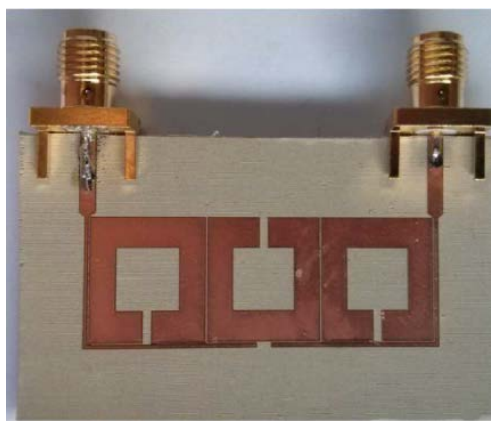

$a$

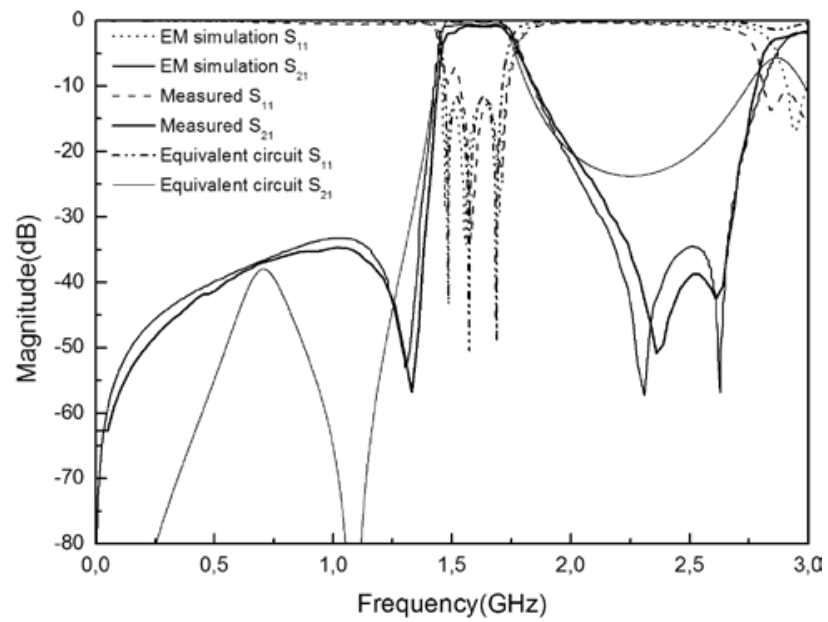

b

Fig. 3 photograph of proposed filter. a Picture of the fabricated prototype. $b$ Comparison between $S_{11}$ and $S_{21}$ measured, electromagnetically simulated and equivalent circuit response.

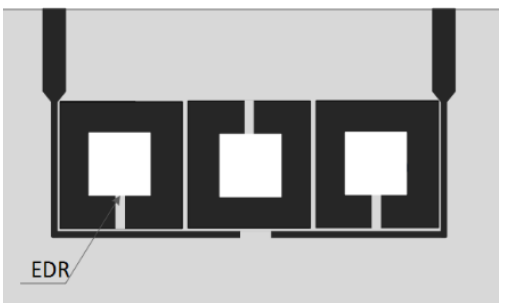

Fig. 4 Layout model of the EDR proposed filter.

Further miniaturization: Further miniaturization has been applied to the design. The miniaturized filter was achieved by increase the value of $\varepsilon_{\mathrm{r}}$ from 45 to 100 , which central frequency proportional to $\varepsilon_{\mathrm{r}}$, shift down from $1.56 \mathrm{GHz}$ to $1.43 \mathrm{GHz}$ respectively. S-parameters comparison between SSR and EDR SSR is depicted in Fig. 5. SSR particle produces a resonant frequency of $1.65 \mathrm{GHz}$, and $\operatorname{EDR}\left(\varepsilon_{\mathrm{r}}=100\right)$ with identical

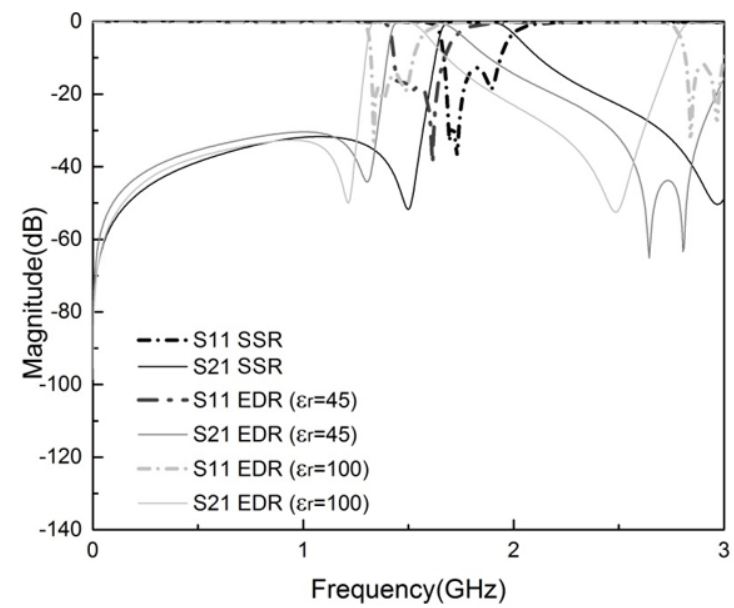

dimensions $1.43 \mathrm{GHz}$. This $14 \%$ of resonant frequency reduction implies an equivalent miniaturization percentage.

Fig. 5 S-parameters comparison between SRR proposed filter and EDR SRR with dielectric constant of 45 and 100.

Conclusion: The proposed equivalent circuit model based on the capacitance coupling transmission line lets to establishing a clear relation between the equivalent circuit model and the layout physical dimensions and, therefore establishing a link between the filter specifications and the layout physical dimensions. The proposed structure points out the possibility of using EDR for further miniaturization. The proposed filter has been fabricated and measured. Further work is under development to improve the utilization of EDR as resonators in planar devices.

B. Moradi, U. Martinez and J. Garcia-Garcia, (Departament d'Enginyeria Electrònica de la Universitat Autònoma de Barcelona, Campus de Bellaterra, 08208 Cerdanyola del Vallés, Barcelona, Spain). Corresponding author E-mail: bahareh.moradi@uab.es.

\section{References}

1 Pendry. J. B., "Negative refraction makes a perfect lens,” Phys. Rev. Lett., vol. 85, no. 18, pp. 3966-3969, 2000.

2 García-García. J, Bonache. J, Gil. I, Martín. F, Velazquez-Ahumada. M.C and Martel. J. "Miniaturized microstrip and CPW filters using coupled metamaterial resonators.” IEEE Transactions on Microwave Theory and Techniques.54 - 6, pp. 2628-2635, 2006.

3 Marques. R, Baena. J, Martel. J, Medina. F, Falcone. F, Sorolla. M, and Martin. F, "Novel small resonant electromagnetic particles for metamaterial and filter design," in Proc. ICEAA, Torino, pp. 439-443, September 2003.

4 Curnmer. A. S, Popa. B. L and Hand. T. H, "Q-based design equations and loss limits for resonant metamaterials and experimental validation,” IEEE Ant. Wireless Prop. Lett, vol. 56, no. 1, pp. 127-132, 2008.

5 García-García. J, Martín. F, Baena. J. D, Marqués. R and Jelinek. L, "On the resonances and polarizabilities of split ring resonators," J. Appl. Phys., vol. 98, p. 033103, 2005.

6 J. García-García. J, Martín F, Falcone. F, Bonache. J, Gil. I, Amat. E, Lopetegi. T, Laso. M.AG,Iturmendi. J.A.M, Sorolla. M and Marqués. $\mathrm{R}$, "Microwave Filters with Improved Stop band based on SubWavelength Resonators,” IEEE Transactions on Microwave Theory and Techniques.53 - 6, pp 1997-2006, 2005.

7 Hong. J and Lancaster. M.L., "Microstrip Filters for RF/Microwave Applications”, John Willey \& Sons, Inc., New York, 2001.

8 Moradi. B, Martinez. U, Ymbern. O, Martinez. C, Alonso. J and Garcia. J "New approach to Electronic Band Gap filtering structures combining

Microstrip and Dielectric Resonators” Asia-Pacific Microw. Conf. Proc. (APMC), Korea, pp 417-419, November 2013. 